\title{
AVALIAÇÃO DE ACESSOS DE MILHO CRIOULO COLETADOS NA REGIÃO CENTRAL DO BRASIL
}

\author{
RAMIRO VILELA DE ANDRADE ${ }^{1}$, MANOEL XAVIER DOS SANTOS ${ }^{1}$, ALEXANDRE DA SILVA \\ FERREIRA $^{1}$, ANTÔNIO CARLOS DE OLIVEIRA ${ }^{1}$
}

\author{
${ }^{1}$ Pesquisadores, Embrapa Milho e Sorgo. Caixa Postal 151, CEP: 35701-970 Sete Lagoas, MG. E-mail: \\ ramiro@cnpms.embrapa.br (autor para correspondência)
}

Revista Brasileira de Milho e Sorgo, v.1, n.2, p.67-74, 2002

\begin{abstract}
RESUMO - A coleção de germoplasma de milho do Brasil é uma das maiores do mundo, constituída de 3.816 acessos armazenados no Banco Ativo de Germoplasma da Embrapa Milho e Sorgo, em Sete Lagoas, MG. Uma das principais causas que dificultam o uso do germoplasma é a pouca informação disponível. Visando ampliar a quantidade e a qualidade de informações úteis para serem utilizadas nos programas de melhoramento, a Embrapa Milho e Sorgo vem desenvolvendo um trabalho sistemático, objetivando caracterizar e avaliar a coleção existente. Com esse enfoque, em 1984, foi publicado o primeiro catálogo brasileiro sobre germoplasma de milho, com a aplicação de 26 descritores em 282 acessos. Em 1986, iniciaram-se os trabalhos pioneiros para a caracterização e a avaliação do germoplasma da América Latina e do Caribe, através do Programa Latino-Americano de Milho (LAMP), com a participação do Brasil na avaliação e caracterização de 1.715 acessos, distribuídos em três regiões ecogeográficas (Sul, Central e Nordeste) e com a publicação de um catálogo correspondente às etapas $1 \mathrm{e} 2$ daquele programa. Em 1996, iniciaram-se novos estudos para implantação de uma segunda etapa do programa LAMP (LAMP II) visando caracterizar e avaliar outros acessos de milho. Assim, no ano agrícola de 1998/99, iniciaram-se os trabalhos para a caracterização de 324 acessos, com a implantação de quatro ensaios em Sete Lagoas, MG, utilizando o delineamento em látice 9x9, com uma testemunha em cada bloco e duas repetições. Foram constituídos grupos homogêneos dentro de cada tipo de endosperma: doce, amiláceo, pipoca, dentado, semidentado, duro e semiduro, utilizando-se o "método do vizinho mais próximo". A dissimilaridade entre os acessos foi determinada pela distância Euclidiana média entre os acessos, tomando-se as características de florescimento masculino e feminino, altura de planta, altura de espiga, planta quebrada e acamada, estande final, prolificidade, peso de espiga e peso de grão na espiga. Os resultados evidenciaram uma grande variabilidade fenotípica entre os acessos para todas as características estudadas, podendo-se selecionar acessos tanto para melhoramento intrapopulacional quanto para a formação de novos compostos.
\end{abstract}

Palavras-chave: Germoplasma, Zea mays L., caracterização, tipo de endosperma, agrupamentos.

\section{EVALUATION OF MAIZE LAND RACESACCESSION COLLECTED INTHE CENTRAL REGION OF BRAZIL.}

\begin{abstract}
The Brazilian maize germplasm collection is one of the greatest in the world with 3,816 accessions preserved in the Active Maize Germplasm Bank of Embrapa Maize and Sorghum Research Center in Sete Lagoas, Minas Gerais, Brazil. One of the main reasons that
\end{abstract}


difficult its use is the lack of information about this germplasm. To increase the amount and the quality of germplasm information, the Embrapa Maize and Sorghum Research Center has been doing a continuous work to characterize and evaluate this maize collection. Within this scope, in 1984, it was published the first catalogue of maize germplasm with 26 descriptors of 282 accessions. In 1986 it was initiated of the program "Latin American of Maize" ( LAMP) to characterize and evaluate the maize germplasm. At that time, 1,715 accessions from Brazil were characterized and evaluated using three geographic regions which resulted in the publication of a catalogue. In 1996, a new LAMP program began ( LAMP II) with the objective of characterizing and evaluating other maize germplasm not considered in the first stage. So, in the 1988/89 season year was established in Sete Lagoas, Minas Gerais, one experiment using 324 accessions of land race germplasm, mostly collected in central Brazil. The experimental design was a 9x9 lattice with four trials and one check within blocks with two replications. The homogeneous groups were classified according to their endosperm type ( sweet, floury, pop, dent, semident, flint and semiflint). The dissimilarities among groups were determined using the Euclidean average distance method, according to the characteristics of male and female flowering, plant height, ear height, stalk and root lodging, final stand, prolificity, ear weight, and grain weight. The results showed high phenotipic variation among accessions for all characteristics, and based on that, it was possible to select accessions to improve intrapopulation varieties and composites formation.

Key words: Germplasm, Zea mays $L$., characterization, endosperm type, clusters

A coleção de germoplasma de milho do Brasil é uma das maiores do mundo, conservada nos bancos de germoplasma da Embrapa e do Cimmyt (Abadie et al. 2000). O Banco Ativo de Germoplasma de Milho está localizado na Embrapa Milho e Sorgo, em Sete Lagoas, MG, onde atualmente encontram-se armazenados 3.816 acessos, sendo $81,3 \%$ de variedades autóctones, 7,6\% de acessos introduzidos de outros países, $5,8 \%$ de variedades melhoradas e 5,3\% de compostos raciais formados a partir das variedades autóctones.

A importância da caracterização e da avaliação do germoplasma é apontada por vários autores (Frankel, 1987; Sloten, 1987; Giacometti, 1988; Valls 1988; Taba et al. 1998).

Segundo Hoyt (1992), os programas de melhoramento, de maneira geral, estão trabalhando com uma variabilidade genética muito estreita, devido a uma série de dificuldades advindas do uso do germoplasma per se. No caso do milho, somente $2 \%$ do germoplasma existente no mundo foi ou está sendo usado pela pesquisa (América,1986). No Brasil, Nass et al. (1993) constataram que a utilização regular do germoplasma de milho pelos melhoristas, nas últimas décadas, foi aproximadamente de 14\%. Duas razões principais são apontadas para a baixa utilização do germoplasma no Brasil: a) falta de adaptação dos genótipos (Miranda Filho, 1992); b) poucas informações relacionadas às características de valor agronômico do germoplasma (Nass et al. 1993).

A experiência tem mostrado, entretanto, que, para as regiões de clima tropical, tem-se obtido sucesso em utilizar esses acessos tanto para uso per se após o melhoramento intrapopulacional quanto para a extração de linhagens para a produção de híbridos (Vasal et al., 1994). Miranda Filho (1974), Paterniani (1990) e Santos et al. (1998) relataram exemplos em que o germoplasma exótico foi introduzido para a redução do porte da planta e para ampliar a variabilidade genética, bem como na utilização de germoplasma tuxpeño na formação de 
variedades melhoradas altamente produtivas e com ampla adaptação.

Desde a sua criação, em 1976, o Banco Ativo de Germoplasma de Milho (BAG) vem desenvolvendo atividades objetivando caracterizar e avaliar o germoplasma existente, contribuindo para aumentar a quantidade e a qualidade de informações úteis para uso em programas de melhoramento. Após anos de trabalho, foi publicado o primeiro catálogo de milho, com a aplicação de 26 descritores morfológicos em 282 acessos (Embrapa, 1984).

Em 1986, iniciaram-se os trabalhos pioneiros com o Programa Latino-Americano de Milho (LAMP), com a participação de 12 países da América Latina e do Caribe, visando ampliar as informações de 12.000 acessos para uso em programa de melhoramento (Salhuana et al. 1991). O Brasil participou do programa, com a caracterização e avaliação de 1.715 acessos em três regiões ecogeográficas do País (Sul, Central e Nordeste), com a publicação de um catálogo referente às etapas 1 e 2, que consistiram na caracterização e avaliação dos acessos durante dois anos consecutivos (Taba et al. 1998).

Em 1996, iniciaram-se os estudos para a implantação de uma nova fase do LAMP, denominada LAMP II, visando à caracterização e à avaliação do germoplasma existente no BAG milho não avaliado na primeira fase.

Com esse enfoque, foram utilizados 324 acessos, na sua maioria originários das regiões $\mathrm{Su}-$ deste e Centro-Oeste, que foram classificados de acordo com o tipo de endosperma, tendo-se por objetivo conhecer a variabilidade dos acessos, bem como constituir grupos homogêneos entre eles, para cada tipo de endosperma.

\section{Material e Métodos}

O ensaio foi conduzido no ano agrícola de 1998/99, na Embrapa Milho e Sorgo, em Sete
Lagoas, $\mathrm{MG}$, com as coordenadas geográficas: latitude $19^{\circ} 28^{\prime} 00^{\prime}$ ' S, longitude $44^{\circ} 15^{\prime} 08^{\prime}$ " W, altitude de $732 \mathrm{~m}$, precipitação média anual de $1.351 \mathrm{~mm}$ e temperatura média anual de $22,6^{\circ} \mathrm{C}$. O clima da região, segundo Koeppen, é do tipo AW-clima de savana, com inverno seco.

Os 324 acessos de milho, na sua maioria constituídos de variedades autóctones, foram avaliados em quatro ensaios e o delineamento experimental utilizado foi em látice $9 \times 9$, com duas repetições. A variedade BR 106, de porte mediano, textura do endosperma semidentado, altamente cultivada nas regiões Sudeste e Centro-Oeste, foi utilizada como testemunha, com o objetivo de viabilizar a análise conjunta dos quatro ensaios. A parcela foi formada por duas linhas de 5,0 m, espaçadas de $0,90 \mathrm{~m}$.

A semeadura foi realizada em meados de outubro de 1998, em solo de cerrado amarelo distrófico e a colheita, no início de abril. A análise de fertilidade do solo apresentou as seguintes características: $\mathrm{pH}-5,9$; saturação de alumínio- 0,0 centimol de carga $\mathrm{dm}^{-3}\left(\mathrm{CMDc} \mathrm{dm}^{-3}\right)$; cálcio-5,0 CMDc $\mathrm{dm}^{-3}$; magnésio-0,9 $\mathrm{CMDc} \mathrm{dm}^{-3}$; potássio140 ppm; fósforo-11 ppm ; matéria orgânica- 3,9 decagramas kg-1 (DAG kg-1).

A adubação de semeadura foi de $15 \mathrm{~kg}$ de nitrogênio, $80 \mathrm{~kg}$ de fósforo, $50 \mathrm{~kg}$ de potássio e $1,5 \mathrm{~kg}$ de zinco por hectare. A adubação nitrogenada de cobertura foi feita aos 35 dias, à base de $30 \mathrm{~kg}$ de $\mathrm{N} \mathrm{ha}^{-1}$, na forma de uréia.

Foram tomados dados por parcela de dez plantas e de dez espigas (Embrapa, 1984) das seguintes características: florescimento masculino, florescimento feminino, altura de planta, altura de espiga, tipo de endosperma e cor do grão. Avaliouse também a porcentagem de plantas quebradas e acamadas por parcela, a prolificidade, o peso de espigas e de grãos e as reações dos acessos às doenças ocorridas (ferrugem, Puccinia polysora; mancha 
por phaeosphaeria, Phaeosphaeria maydis; helmintosporiose, Exerohilum turcicum; enfezamento, Phytoplasma e Spiroplasma). Para a avaliação da severidade das doenças, utilizou-se a seguinte escala de notas: 1- ausência de doença; 2presença de doença em poucas plantas; 3 - ocorrência da doença em mais de $50 \%$ das plantas, porém com baixa severidade; 4- ocorrência da doença em $100 \%$ das plantas, com até $25 \%$ da área foliar afetada; 5- doença severa, semelhante ao item 4, porém com mais de $25 \%$ da área foliar afetada. Para o enfezamento, os dados foram expressos em porcentagem de plantas com sintomas.

Dentro de cada tipo de endosperma, os acessos foram agrupados de modo a formar grupos homogêneos em relação às características relatadas anteriormente, com exceção da coloração do grão e doenças, que foram expressas em valores mínimos e máximos. Os acessos foram classificados de acordo com os seguintes tipo de endosperma: doce, amiláceo, pipoca, dentado, semidentado, duro e semiduro.

Foram determinadas as dissimilaridades entre os acessos dentro de cada grupo (tipo de endosperma) utilizando-se a distância Euclidiana média, através do "método do vizinho mais próximo", conforme descrito por Cruz \& Regazzi (1997), tomando-se as características de florescimento masculino e feminino, altura de planta, altura de espiga, planta quebrada e acamada, prolificidade, peso de espiga e de grão.

As análises foram realizadas utilizando-se o procedimento "Proc cluster" do programa SAS (2000).

\section{Resultados e Discussão}

Os agrupamentos por tipo de endosperma, dos acessos que apresentaram características homogêneas, estão apresentados na Tabela 1. Foi observada uma grande variabilidade fenotípica para todos os acessos avaliados. Através do método das distâncias Euclidianas, foram agrupados os acessos que apresentaram grandes similaridades entre si para os sete tipos de endosperma estudados, a saber:

Grupo 1 - DOCE - Cinco acessos foram classificados para essa característica e separadas em quatro agrupamentos. O agrupamento 1, constituído de dois acessos, diferenciou-se dos demais principalmente por apresentar florescimento feminino tardio (66 dias), maior altura de planta $(336,0 \mathrm{~cm}) \mathrm{e}$ de espiga $(210,8 \mathrm{~cm})$ e maior peso de espiga $(116,2$ g) e de grãos $(86,8 \mathrm{~g})$, sendo os grãos de coloração amarela. Na avaliação para enfermidades, o agrupamento apresentou-se como resistente à ferrugem e ao enfezamento. O agrupamento 2, constituído de apenas um acesso, apresentou características de precocidade, pelo florescimento feminino $(51,5$ dias), índice de prolificidade $(1,1)$ e menor altura de planta $(269,3 \mathrm{~cm})$ e de espigas $(118,2 \mathrm{~cm})$, porém com baixa produtividade $(41,7 \mathrm{~g})$, resistente a helmintosporiose e moderadamente resistente a Phaeosphaeria. Os agrupamentos 3 e 4 apresentaram-se como resistentes à ferrugem, ciclos normais pelo florescimento feminino (58,2 e 60,7 dias) e com alturas intermediárias de planta $(286,5$ e 311,7 $\mathrm{cm})$ e de espiga $(138,2$ e $170,5 \mathrm{~cm})$.

Grupo 2 - AMILÁCEO - Dez acessos foram classificados para essa característica e separados em quatro agrupamentos. O agrupamento 1, constituído de quatro acessos, apresentou-se com características de precocidade, pelo florescimento feminino (55,5 dias), menores alturas de planta $(299,6 \mathrm{~cm})$ e de espiga $(161,1 \mathrm{~cm})$, menor porcentagem de plantas quebradas e acamadas $(12,7 \%)$, maior índice de prolificidade $(1,1)$ e maior produtividade por planta $(77,3 \mathrm{~g})$. Houve grande variação na severidade de todas as doenças ocorridas. $\mathrm{O}$ agrupamento 2, formado por 1 acesso, apresentou características de um material tardio pelo florescimento feminino(78,4 dias), resistentea Phaeosphaeria 
e a Puccinia polysora, porém com baixíssima produtividade por planta $(6,4 \mathrm{~g})$, talvez causada pela alta incidência do Exerohilum turcicum. Os demais agrupamentos apresentaram-se com características intermediárias de florescimento, altura de planta $\mathrm{e}$ de espiga, produtividade e também com grande variação de severidade de doenças.

Grupo 3 - PIPOCA - Treze acessos foram incluídos nesse grupo e separados em quatro agrupamentos. $\mathrm{O}$ agrupamento 3 , com quatro acessos, apresentou característica de precocidade, pelo florescimento feminino (52,4 dias) menores alturas de planta $(245,3 \mathrm{~cm})$ e de espiga $(119,7 \mathrm{~cm})$, baixo índice de prolificidade $(0,8)$, baixa produtividade de grãos $(13,9 \mathrm{~g})$, resistência moderada às doenças, com exceção do enfezamento, que não foi avaliado devido ao avançado estágio de desenvolvimento das plantas por ocasião da tomada dos dados. O agrupamento 2, com três acessos, apresentou-se mais tardio, pelo florescimento feminino (65,7 dias), maiores alturas de planta $(356,2 \mathrm{~cm})$ e de espiga $(219,7$ $\mathrm{cm})$, índice de prolificidade $(1,2)$ e de produção de grãos intermediária $(52,0 \mathrm{~g})$. Os agrupamentos $2 \mathrm{e}$ 4, com três e cinco acessos, respectivamente, apresentaram também ciclos tardio a intermediário, pelo florescimento feminino (65,7 e 61,4 dias), alturas de planta de 356,2 e $307,2 \mathrm{~cm}$, porém mais produtivos que os demais agrupamentos (52,0 e 74,1 $\left.\mathrm{g} \mathrm{planta}^{-1}\right)$. Houve grande variação de reação dos acessos em relação às doenças, variando de resistente a suscetível.

Grupo 4 - DENTADO - Noventa e nove acessos foram classificados nesse grupo, distribuídos em dez agrupamentos. Nos agrupamentos 2, 3 e 4 , estão representados cerca de $87 \%$ dos acessos, mostrando a uniformidade desse tipo de endosperma quanto às características morfológicas estudadas. Os acessos, apresentaram características de ciclo intermediário, pelo florescimento feminino (61,9 a 67,6 dias), altura de planta de 326,8 a
$375,6 \mathrm{~cm}$, boa produtividade (80,1 a 118,4 g) e grande variação de resultados quanto às doenças avaliadas. $\mathrm{O}$ agrupamento 5, com três acessos, apresentou características de precocidade, pelo florescimento masculino (46,2 dias), menores alturas de planta $(258,5 \mathrm{~cm})$ e de espiga $(112,8 \mathrm{~cm})$ e menor porcentagem de plantas quebradas e acamadas $(2,3 \%)$. Com relação à cor do grão, esse agrupamento apresentou coloração amarela, amareloalaranjada e alaranjada. Essas características são desejáveis para os programas de melhoramento, podendo ser exploradas através dos diferentes agrupamentos, de acordo com o tipo de endosperma (Centro,1998). O agrupamento 1, com quatro acessos, apresentou ciclo tardio, pelo florescimento feminino (72,1 dias), maiores alturas de planta (365,2 $\mathrm{cm})$ e de espiga $(225,1 \mathrm{~cm})$ produtividade intermediária $(66,4 \mathrm{~g})$, porém com grande variação na severidade de doenças. Já o agrupamento 10 , mostrou-se tardio, pelo florescimento feminino $(76,8$ dias), com altura elevada $(380,8 \mathrm{~cm})$ e com alta porcentagem de plantas quebradas e acamadas (45,9\%). Apresentou também baixa produção de grãos por espiga (33,7 g), provavelmente causada pela alta incidência da helmintosporiose e mancha por Phaeosphaeria.

Grupo 5 - SEMIDENTADO - O maior número dos acessos foi classificado nesse grupo, ou seja, 106 acessos, sendo que $74 \%$ destes estão concentrados no primeiro e no segundo agrupamentos, o que mostra a maior similaridade dos acessos pertencentes a esse tipo de endosperma. Esses dois agrupamentos apresentaram-se como sendo de florescimento feminino intermediário (64 e 67 dias), plantas e espigas relativamente altas $(329,5$ e 335,8 $\mathrm{cm}, 189,7$ e 200,2 cm, respectivamente), alta porcentagem de plantas quebradas e acamadas $(19,2 \mathrm{e}$ $25,6 \%$ ) e grande variação na coloração dos grãos e na severidade de doenças. $\mathrm{O}$ agrupamento 10 apresentou-se como sendo o mais precoce, pelo 
TABELA 1. Médias de oito características dos grupos homogêneos formados por 324 acessos de milho, de acordo com o tipo de endosperma. Embrapa Milho e Sorgo. 2002

\begin{tabular}{|c|c|c|c|c|c|c|c|c|c|c|c|c|c|c|c|}
\hline \multirow{2}{*}{$\begin{array}{c}\text { Tipo de } \\
\text { Endosperma }\end{array}$} & \multirow[t]{2}{*}{ AGP } & \multirow[t]{2}{*}{$\mathrm{NAC}$} & \multirow[t]{2}{*}{ FLM } & \multirow[t]{2}{*}{ FLF } & \multirow[t]{2}{*}{ AP } & \multirow[t]{2}{*}{$\mathbf{A E}$} & \multirow[t]{2}{*}{ PRO } & \multirow[t]{2}{*}{ PQAC } & \multirow[t]{2}{*}{ PESP } & \multirow[t]{2}{*}{ PGP } & \multirow[t]{2}{*}{$\mathrm{CGR}^{2}$} & \multicolumn{4}{|c|}{ Doenças (Variação minimo-máximo) } \\
\hline & & & & & & & & & & & & $\mathrm{Ph}^{\mathrm{b}}$ & FP $^{\mathrm{b}}$ & HEL $^{b}$ & ENF $\%$ \\
\hline \multirow{4}{*}{ Doce } & 1 & 2 & 63,0 & 66,0 & 336,0 & 210,8 & 1,2 & 14,9 & 116,2 & 86,8 & 2 & $3,0-3,5$ & $1,0-2,0$ & $3,0-3,5$ & $0,0-2,2$ \\
\hline & 2 & 1 & 49,0 & 51,5 & 269,3 & 118,2 & 1,1 & 15,5 & 45,9 & 41,7 & 7 & 3,0 & 3,5 & 2,5 & 2,4 \\
\hline & 3 & 1 & 56,9 & 58,2 & 286,5 & 138,2 & 1,0 & 13,4 & 105,8 & 86,3 & 16 & 4,0 & 1,0 & 4,0 & 6,9 \\
\hline & 4 & 1 & 58,4 & 60,7 & 311,7 & 170,5 & 13 & 24,2 & 85,5 & 70,6 & 16 & 3,5 & 2,5 & 4,0 & 10,1 \\
\hline \multirow{4}{*}{ Amiláceo } & 1 & 4 & 53,6 & 55,4 & 299,6 & 161,1 & 1,1 & 12,7 & 97,7 & 77,3 & $2-4$ & $2,5-4,5$ & $2,5-3,5$ & $2,0-4,0$ & $0,0-31,0$ \\
\hline & 2 & 1 & 73,4 & 78,4 & 353,8 & 256,3 & 0,6 & 14,7 & 6,5 & 6,4 & 7 & 2,5 & 1,0 & 4.5 & 4,4 \\
\hline & 3 & 2 & 63,1 & 66,4 & 373,4 & 230,7 & 1,0 & 29,5 & 49,6 & 40,0 & $2 \cdot 9$ & 3,0 & $3,0-3,5$ & $4,0-4,5$ & $2,2-6,2$ \\
\hline & 4 & 3 & 62.3 & 65,5 & 338,0 & 181.2 & 0,7 & 17,8 & 36,4 & 28,6 & $3-9$ & $4,0-4.5$ & $3,0-3,5$ & 4.0 & $6,5-1$ \\
\hline \multirow{4}{*}{ Pipeca } & 1 & 1 & 62,0 & 65,5 & 278,5 & 158,2 & 1,3 & 5,6 & 42,7 & 31,6 & 4 & 4,0 & 3,5 & 4,0 & 4,6 \\
\hline & 2 & 3 & 64,1 & 65,7 & 356,2 & 219,7 & 1,2 & 17,2 & 65,9 & 52,0 & 7.8 & $2,0-4,0$ & $3,5-4,0$ & $3,5=4,0$ & $0,0-10,0$ \\
\hline & 3 & 4 & 49,7 & 52,4 & 245,3 & 119.7 & 0,8 & 7,8 & 17,6 & 13,9 & $3-7-8$ & 3,5 & $3,0-3,5$ & 3,5 & - \\
\hline & 4 & 5 & 59.5 & 61,4 & 307,2 & 184,6 & 1,4 & 17,0 & 915 & 74,1 & 3. $7 \cdot 8$ & $3,0-4,0$ & $1,5-4,0$ & $3,5=4,0$ & $0,0-6,7$ \\
\hline & 1 & 4 & 68.2 & 72,1 & 365,2 & 225,1 & 0,6 & 35,6 & 66,4 & 48,7 & $1=2$ & $3,0-4,0$ & $2,0-3,5$ & $2,5=4,0$ & $0,0-11,0$ \\
\hline & 2 & 22 & 63,4 & 66,0 & 375,6 & 229,9 & 1,0 & 15,2 & 148,0 & 118,4 & $1-2-3-4-6-7-12-15$ & $2,5-4,5$ & $1,0-4,0$ & $2,5-4,0$ & $0,0-6,3$ \\
\hline & 3 & 32 & 59,8 & 61,9 & 326,2 & 181,1 & 1,0 & 18,8 & 131.5 & 105.7 & $2-3-4-6$ & $3,0-5,0$ & $1,5-3,5$ & $2,0-4,0$ & $0,0-13,0$ \\
\hline & 4 & 32 & 64,7 & 67,6 & 336,8 & 202,8 & 0,8 & 25,4 & 103,2 & 80,1 & $2-3-6$ & $3,0-4,5$ & $1,0-4,0$ & $3,0-4,0$ & $0,0-15,0$ \\
\hline & 5 & 3 & 46,2 & 50,6 & 258,5 & 112,8 & 1,0 & 2,3 & 112,6 & 91,1 & $2-6-7$ & $4,0-4,5$ & $2,0-4,5$ & $2,5-3,5$ & $4,2-6,4$ \\
\hline Dentado & 6 & 2 & 61,7 & 64,3 & 338,9 & 197,4 & 0,9 & 19,4 & 123,7 & 98,4 & 1.14 & $3,0-4,0$ & $2,5-3,0$ & $4,01-4,5$ & $4,1-6,7$ \\
\hline & 7 & 1 & 60,1 & 62.2 & 270.2 & 120,7 & 0.8 & 8.2 & 66,2 & 52,1 & 2 & 3.5 & 1,0 & 2,0 & 0.0 \\
\hline & 8 & 1 & 54,0 & 58,0 & 314,2 & 144,0 & 0,7 & 18,9 & 14,1 & 5,7 & 10 & 4,0 & 4,0 & 3,5 & 0,0 \\
\hline & 9 & 1 & 61.4 & 62,7 & 352,8 & 205,9 & 1,4 & 19,6 & 63,4 & 48,6 & 7 & 2,5 & 3,5 & 4,0 & 2,3 \\
\hline & 10 & 1 & 71,2 & 76,8 & 380,8 & 246,7 & 0,3 & 46,0 & 33,4 & 22,1 & 1 & 4,0 & 2,5 & 4,0 & 0,0 \\
\hline & 1 & 48 & 61,4 & 64,0 & 329,5 & 189,7 & 1,02 & 19,2 & 133,5 & 106,3 & $1-2-3-4-5-6-7-8$ & $2,5-4,5$ & $1,5-4,0$ & $2,0-4,5$ & $0,0-22,1$ \\
\hline & 2 & 31 & 64,4 & 67,0 & 335,8 & 200,2 & 0,84 & 25,6 & 101,0 & 79,5 & $1-2-3-4-5-6$ & $2,5-4,5$ & $1,5-4,5$ & $2,5-4,5$ & $0,0-15,0$ \\
\hline & 3 & 6 & 54,7 & 56,1 & 303,3 & 142,3 & 1,0 & 10,2 & 134,0 & 108,1 & $2-3-6=8$ & $3,0-4,5$ & $1,5-3,0$ & $2,0-4,0$ & $0,0-6,2$ \\
\hline & 4 & 7 & 65,8 & 68,6 & 379,1 & 243,4 & 0,9 & 23,1 & 116,8 & 84,0 & $1-2-3-6$ & $2,5-4,0$ & $1,0-4,0$ & $3,5-4,0$ & $0,0-8,6$ \\
\hline & 5 & 2 & 63,0 & 65,2 & 300,5 & 162,0 & 1.4 & 7,8 & 151,0 & 121,1 & $2 \cdot 3$ & $2,5-4,0$ & $1,5-2,5$ & $2,0-3,0$ & $0,0-5,7$ \\
\hline & 6 & 3 & 71,1 & 75,2 & 355,5 & 227,9 & 0,8 & 38,5 & 100,8 & 77,7 & $1-2-3$ & $1,5-4,5$ & $1,5-3,5$ & $2,5-3,5$ & $2,1-9,0$ \\
\hline Semidentado & 7 & 2 & 67.8 & 72,0 & 350,0 & 206,8 & 0,5 & 42,5 & 64,0 & 48.4 & 3 & $3,5-4,0$ & $2,5-3,0$ & $2,5-4,0$ & $0,0-6,8$ \\
\hline & 8 & 2 & 46,6 & 49,7 & 250,8 & 104,0 & 0,7 & 3,1 & 30,8 & 23,3 & $2-5$ & $2,5-3,5$ & $3,0-3,5$ & 3.5 & $-4,3$ \\
\hline & 9 & 1 & 62,8 & 64,0 & 384,2 & 242,6 & 1,1 & 12,2 & 145,6 & 118,2 & 4 & 4,0 & 3,5 & 4,0 & 0,0 \\
\hline & 10 & 1 & 46,6 & 49,2 & 237,8 & 102,2 & 1,1 & 1,0 & 108,2 & 88,6 & 6 & 3,5 & 4,0 & - & 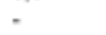 \\
\hline & 11 & 1 & 50,8 & 55,6 & 206,0 & 102,2 & 0.7 & 2,6 & 1,8 & 0.4 & I & 3.5 & 3,0 & - & 0,0 \\
\hline & 12 & 1 & 49,2 & 52,0 & 302,4 & 146,4 & 1,0 & 5,0 & 44,0 & 41,6 & 1 & 4,0 & 2,0 & - & 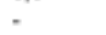 \\
\hline & 13 & 1 & 60.5 & 62,0 & 279,2 & 152,0 & 0.9 & 8.4 & 67.1 & 58,6 & 4 & 4,0 & 4,0 & 3.5 & 15.3 \\
\hline & 1 & 22 & 62,3 & 64,8 & 329,0 & 189,0 & 1.0 & 21,2 & 114,1 & 90,3 & $6-7-8-9-11-13$ & $3,0-4,5$ & $1,5-3,5$ & $2,0-4,0$ & $0,0-23,0$ \\
\hline & 2 & 15 & 48,6 & 51,0 & 263,4 & 120,9 & 0,8 & 14,0 & 43,8 & 35,2 & $2-7$ & $2,5-4,0$ & $2,0-4,0$ & $=$ & $=$ \\
\hline Duro & 3 & 3 & 61,6 & 63,9 & 352,4 & 205,3 & 1,3 & 25,3 & 135,0 & 107,4 & 6- $7-13$ & $3,5-4,5$ & $3,0-4,0$ & $3,0-4,0$ & $0,0-2,3$ \\
\hline & 4 & 4 & 64,0 & 66,5 & 350,6 & 219,4 & 0,9 & 28,2 & 87,6 & 66,1 & 3. 7.8 & $3,0-4,0$ & $2,0-4,0$ & $3,5-4,5$ & $0,0-8,5$ \\
\hline & 5 & 2 & 45.5 & 47,3 & 229,0 & 83.5 & 1.0 & 5.4 & 93.5 & 75,7 & 7 & $3,0-4,0$ & 3,0 & $2,0-$ & $2,1 \cdots$ \\
\hline & 1 & 4 & 67,7 & 70,6 & 353,8 & 211,3 & 0,7 & 35,4 & 81.8 & 60,8 & $2-3-7-8$ & $3,0-4,0$ & $2,5-3,0$ & $3,5-4,0$ & $0,0-6,1$ \\
\hline & 2 & 6 & 46,9 & 48,8 & 259,6 & 120,9 & 0,9 & 7,6 & 49,5 & 42,0 & $1-2=3-6=7$ & $3,5-4,0$ & $3,0-4,5$ & $2,5=$ & $0,0=$ \\
\hline & 3 & 15 & 60.5 & 62,6 & 316,4 & 176,7 & 1,1 & 14,4 & 133,7 & 107,9 & $3-6-7-8$ & $3,0-4,5$ & $1,5-3,5$ & $2,5-4,0$ & $0,0-13,0$ \\
\hline & 4 & 16 & 63,2 & 65,7 & 330,7 & 194,2 & 0,9 & 23,9 & 113,9 & 89,7 & $3-6-7-8$ & $3,0-4,0$ & $1,5-3,5$ & $2,5-4,0$ & $0,0=19,0$ \\
\hline Semiduro & 5 & 1 & 64,8 & 66,2 & 366,0 & 207,4 & 0,9 & 6,9 & 162,1 & 124,6 & 1 & 2,5 & 4,0 & 3,5 & 0,0 \\
\hline & 6 & 1 & 64,6 & 65.9 & 383,5 & 243,4 & 1,1 & 23,8 & 95,6 & 77,3 & 13 & 4,0 & 2,5 & 3,5 & 0,0 \\
\hline & 7 & 1 & 56.9 & 59,8 & 270,1 & 137,2 & 0,9 & 26,9 & 92.5 & 71,7 & 6 & 4,0 & 2,5 & 3.5 & 8,9 \\
\hline & 8 & 1 & 42,8 & 44,1 & 206,5 & 69.7 & 1,0 & 9,17 & 95,3 & 78.8 & 1 & 3,5 & 2.5 & 2,5 & - \\
\hline
\end{tabular}

\section{AGP Agrupamento}

FLM Florescimento masculino(dias)

AP Altura de planta(cm)

PQAC Plantas quebradas e acamadas(\%)

$\mathrm{Ph}^{\mathrm{a}} \quad$ Lesão por Phaeosphaeria maydis

Enf Lesão por Mycoplasma e Spiroplasma (\%)

PRO Prolificidade pelo $n^{\circ}$ de espiga

$\mathrm{CGR}^{\mathrm{b}} \quad$ Cor do grão
NAC $\mathrm{N}^{\circ}$ de acessos do agrupamento

FLF Florescimento feminino(dias)

AE Altura espiga $(\mathrm{cm})$

PESP Peso de espiga $(\mathrm{g})$

FPa $^{a}$ Lesão por Puccinia polysora

$\mathrm{Hel}^{\mathrm{a}}$ Lesão por Exerohilum turcicum

PGP Peso de grão(g)

a1 - branco; 2 - amarelo; 3 - amarelo e branco; - 4 amarelo e roxo; 5 - amarelo e vermelho; 6 - amarelo/ alaranjado; 7 - alaranjado; 8 - branco e alaranjado; 9 - alaranjado e preto; 10 - branco e roxo; 11 - alaranjado e roxo; 12 - vermelho; 13 - roxo; 14 - branco; preto-alaranjado; 15 - branco e preto; 16 - preto e amarelo. b1 a 2,5 - resistente; 3 a 3,5 - moderadamente resistente; 4 a 5 - susceptível. 
florescimento feminino (49,2 dias), menores alturas de planta $(237,8 \mathrm{~cm})$ e de espiga $(102,2 \mathrm{~cm})$, relativamente prolífico $(1,08)$, resistente ao acamamento e ao quebramento $(0,99 \%)$, média produtividade de grãos $(88,6 \mathrm{~g})$ e moderadamente resistente à mancha por Phaeosphaeria. Os acessos do agrupamento 6 foram os mais tardios, pelo florescimento feminino (75,2 dias), com plantas e espigas relativamente altas $(355,5 \mathrm{~cm}$ e $227,9 \mathrm{~cm})$, média produtividade $(100,8 \mathrm{~g})$ e resistente a moderadamente resistente à ferrugem e à helmintosporiose. Os acessos do agrupamento 5 foram os que apresentaram maiores produtividades de espiga $(151,0 \mathrm{~g})$ e de grãos $(121,1 \mathrm{~g})$ e maior índice de prolificidade $(1,39)$, sendo as plantas resistentes a Puccinia polysora e à helmintosporiose.

Grupo 6 - DURO - Constituído de 46 acessos, sendo que $80 \%$ desses estão concentrados nos agrupamentos 1 e 2 . Os acessos do agrupamento 1 apresentaram valores medianos para a maioria das características avaliadas, grande variação na coloração do grão e com nível de resistência à ferrugem variando de moderado a alto. Os acessos do agrupamento 5 apresentaram características de maior precocidade, pelo florescimento feminino $(47,3$ dias), índice de prolificidade $(1,0)$, menores alturas de planta $(229,0 \mathrm{~cm})$ e de espiga $(83,5 \mathrm{~cm})$, baixa taxa de quebramento e acamamento $(5,4 \%)$ e níveis de resistência alta à helmintosporiose e moderada à ferrugem.

Grupo 7 - SEMIDURO - Quarenta e cinco acessos foram classificados para esse tipo de endosperma, distribuídos em oito agrupamentos. À semelhança dos milhos duros, não houve acessos com características muito tardias para esse tipo de milho. O agrupamento 3, com 15 acessos, apresentou características intermediárias para o florescimento feminino, de 62,6 dias, média de altura de plantas de $316,4 \mathrm{~cm}$, de espiga de $176,6 \mathrm{~cm}$ e porcentagem de plantas quebradas e acamadas intermediária, de $14,4 \%$, produtividade de espigas de $107,9 \mathrm{~g} \mathrm{e}$ índice de prolificidade de 1,1. Foi observada grande variação na incidência de doenças, com exceção da ferrugem, uma vez que os acessos foram classificados como resistentes a moderadamente resistentes. O agrupamento 5, embora constituído de um único acesso, apresentou a menor porcentagem de plantas quebradas e acamadas $(6,96)$, maior produtividade $(162,12 \mathrm{~g})$, resistente a Phaeosphaeria e ao enfezamento e resistência moderada à helmintosporiose. Já o agrupamento 8 foi o que apresentou maior precocidade, pelo florescimento feminino (44,1 dias), menores alturas de planta $(206,5 \mathrm{~cm})$ e de espiga $(69,7 \mathrm{~cm})$, resistente à ferrugem e à helmintosporiose e com resistência moderada a Phaeosphaeria.

\section{Conclusão}

Foi observada grande variabilidade fenotípica para todas as características estudadas, podendo-se selecionar acessos tanto para melhoramento quanto para a formação de novos compostos.

\section{Agradecimentos}

Ao assistente de operações José da Silva pela contribuição na condução dos ensaios e tomada de dados.

\section{Literatura Citada}

ABADIE, T; CORDEIRO, C.M.T.; ANDRADE, R.V; MAGALHÃES, J.R; PARENTONI, S.N. A coleção nuclear de milho no Brasil. In: UDRY, C. V.; DUARTE, W. Uma história brasileira do mi-

lho no Brasil: o valor dos recursos genéticos. Brasília: Paralelo 15, 2000. p 65-78

AMÉRICA Latina y Sus Recursos Abundantes de Alimentos para El Futuro. Jonhston: Pioneer Hi-Bred International, 1986.44p. 
CENTRO DE INFORMÁTICA PARA LA INVESTIGACIÓN AGRÍCOLA. Proyecto latinoamericano del maíz (LAMP):informe final. Lima, [1998 ?]. 52p

CRUZ, C. D.; REGAZZI, A. J. Métodos biométricos aplicados ao melhoramento genético. Viçosa: UFV, 1997. 390p

EMBRAPA. Centro Nacional de Recursos Genéticos (Brasília, DF). Catálogo de germoplasma de milho, Zea mays L. Brasília, 1984. 102p.

FRANKEL, O. H. Genetic Resources. The founding gears. Part four: After twenty years. Diversity, Arlington, v.5, n.4, p. 25-27, 1987

GIACOMETTI, D.C. Descritores para caracterização e avaliação de germoplasma. In: ENCONTRO SOBRE RECURSOS GENÉTICOS, 1, 1988, Jaboticabal, SP. Anais ... Jaboticabal: UNESP - FCAV / EMBRAPA - CENARGEN, 1988. p.129-139.

HOYT, E. Conservação dos parentes silvestres das plantas cultivadas.[S.1.] IBPGR / IUCN / WWF / EMBRAPA-CENARGEN/ AddisonWesley Iberoamericana, 1992. 52p.

MIRANDA FILHO, J. B. Cruzamentos dialélicos e síntese de compostos de milho (Zea mays L.) com ênfase na produtividade e no porte da planta. 1974. 116f. Tese (Doutorado) Universidade de São Paulo, Escola Superior de Agricultura Luiz de Queiroz, Piracicaba.

MIRANDA FILHO, J. H. Exotic germplasm introduced in a Brazilian maize breeding program. Revista Brasileira de Genética, Ribeirão Preto, v. 16, p. 983-988, 1992.

NASS, L.L.; PELLICANO, I.J.; VALOIS, A.C.C. Utilization of genetic resources for maize and soybean breeding in Brazil. Revista Brasileira de Genética, Ribeirão Preto, v.16, n.4, p.983-988, 1993.
PATERNIANI, E. Maize breeding in the tropics. CRC Critical in Plant Science, Boca Raton, v. 9, p.125-154, 1990.

SALHUANA, W.; JONES, Q.; SEVILLA,R. The Latin american Maize Project: Model for rescue and use of irreplaceable germplasm. Diversity, Arlington, v. 7, p.40-42, 1991.

SANTOS, M. X.; CARVALHO, H.W.L.; LEITE, C.E.P.; ANDRADE, R.V.; VASCONCELLOS, C.A. Evaluation and selection of tropical maize (Zea mays L.) accessions in low-fertility soils with phosphorus limitation. Plant Genetic Resources Newsletter, Rome, 113, p.17-21. 1998.

SAS. INSTITUTE INC. SAS/STAT. User guide. Verson 8.0 Cary, 2000 p.892-1686.

SLOTEN, D. H van. The role of curators, breeders and other uses of germplasm In: IBPGR. Characterization and evaluation of crop genetic resources. Rome, 1987. p.3-8.

TABA,.S.; DIAZ, J.; FRANCO, J. A core subset of LAMP (Latin American Maize Project)- from the evaluation trials at stage 1 . Mexico-DF: CIMMYT, 1998. 427p.

VALLS, J.F.M. Caracterização morfológica, reprodutiva e bioquímica de germoplasma vegetal. In: ENCONTRO SOBRE RECURSOS GENÉTICOS, 1, 1988, Jaboticabal. Anais... Jaboticabal: UNESP - FCAV / EMBRAPA - CENARGEN, 1988. p.106-128.

VASAL, S. K.; CORDOVA, H.; BJARNASON, M; PIXLEY, K.; LOTHORP, J.; EDMEADES, G.; LAFITTE, H. R.; MHIM, J.A.;JEWELL, D. C. Utilization of germplasm bank materials: the Cimmyt maize program. In: TABA, S. (Ed) The Cimmyt maize germplasm bank: genetic resource preservation, regeneration, maintenace and use. Mexico: CIMMYT, 1994. p. 21-42 (Maize Program, Special Report). 